\title{
Drug-induced acute pancreatitis
}

Tracie Kaurich, PharmD

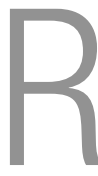

eports of drug-induced acute pancreatitis (AP) have been published since the 1950s, and each year the list of drugs associated with AP increases. There are many etiological risk factors for AP, including a history of alcohol abuse, gallstones, endoscopic retrograde cholangiopancreatography and manometry, trauma or surgical procedures near the pancreas, certain medications, hyperlipidemia, infection, and chronic hypercalcemia $(1,2)$. Knowledge of the true incidence of drug-induced AP is dependent on clinicians excluding other possible causes and reporting the event. It can be difficult to rule out other causes of AP, especially in patients who have multiple comorbidities, use multiple medications, and have potentially unknown underlying risk factors. A retrospective study conducted in Germany concluded that the incidence of drug-induced AP is $1.4 \%$ (3). A national survey performed in Japan in 1999 reported that $1.2 \%$ of all cases of AP were drug induced (2). Drug-induced AP is rare but should not be overlooked in a patient who presents with idiopathic AP.

The exocrine function of the pancreas includes the production of digestive enzymes for release into the gastrointestinal tract $(1,4)$. The acinar cells within the pancreas are responsible for producing the proenzymes, which then are packaged into storage vesicles called zymogens. The zymogens travel through the pancreatic duct and are secreted into the duodenum. Within the duodenum, enterokinase converts trypsinogen to trypsin, and then active trypsin facilitates the conversion of the other pancreatic proenzymes to the active form. AP can occur if there is damage to the acinar cells and/or injury to the pancreatic duct that leads to inappropriate accumulation and activation of proenzymes within the pancreas. The activated pancreatic enzymes digest the cell membranes of the pancreas and activate an inflammatory response, which increases the vascular permeability of the pancreas. Hemorrhage, edema, ischemia, and necrosis can result $(1,4)$. In severe AP, patients progress to systemic inflammatory response syndrome, sepsis, and multiple organ failure (5). About $3 \%$ to $13 \%$ of AP cases progress to chronic pancreatitis (2).

When AP progresses to a severe illness, patients can experience extended hospital stays and increased health care costs. Neoptolemos and colleagues reported that approximately 25\% of patients who develop AP will require intensive care treatment (5). In a retrospective study conducted between January 1992 and December 1996, patients with AP who required intensive care treatment had an average intensive care unit stay of 9 days and an average total hospital stay of 39 days (6). The investigators also concluded that the average overall hospital cost was $\$ 96,891$. Fortunately, AP patients typically regain complete functional ability and can return to employment and other normal activities $(5,6)$.

In previous years, experts created a classification system that addressed the likelihood that certain drugs would be associated with AP, using the categories of definite, probable, and questionable/possible. Over the years, the list of medications associated with AP has increased. Most recently, Badalov and colleagues expanded the classification system to five categories: Ia, Ib, II, III, and IV (7). Classifications are based on the number of case reports, available rechallenge data, consistent latency period, and ability to exclude other causes of AP. Class Ia includes drugs with at least one case report, evidence of a positive rechallenge, and exclusion of other causes of AP. Class Ib is similar to class Ia, but in this class, other causes of AP could not be ruled out. Criteria for class II drugs include at least four case reports with a consistent latency period for at least $75 \%$ of the cases. Class III drugs have at least two case reports but do not have rechallenge data or a consistent latency period. Finally, class IV drugs have one case report without rechallenge data. The Table is a noninclusive list of many medications associated with AP (7-11).

The mechanisms of action for drug-induced AP are based on theories extracted from case reports, case-control studies, animal studies, and other experimental data. In general, some potential mechanisms of action for drug-induced AP include pancreatic duct constriction, cytotoxic and metabolic effects, accumulation of a toxic metabolite or intermediary, and hypersensitivity reactions (12). Other mechanisms for drug-induced AP have been associated with adverse effects of drugs, such as hypertriglyceridemia or chronic hypercalcemia, which are risk factors for AP. More specifically, a potential mechanism of action for angiotensin-converting enzyme (ACE) is a local-

From the Department of Pharmacy Services, Baylor University Medical Center, Dallas, Texas.

Corresponding author: Tracie Kaurich, PharmD, Department of Pharmacy Services, Baylor University Medical Center, 3500 Gaston Avenue, Dallas, Texas 75246 (e-mail: tracieka@BaylorHealth.edu). 
Table. Drugs and drug classes associated with acute pancreatitis*

\begin{tabular}{|c|c|c|}
\hline ACE inhibitors & Estrogens & Pentamidine \\
\hline Acetaminophen & Ethacrynic acids & Pergolide \\
\hline Adrenocorticotrophic & Exenatide & Phenolphthalein \\
\hline hormones & Ezetimibe & Pilocarpine \\
\hline Alendronate & Fibrates & Prazosin \\
\hline All-trans-retinoic acid & Finasteride & Procainamide \\
\hline Alpha-methyldopa & Fluoroquinolones & Propofol \\
\hline Aminosalicylates & 5-Fluorouracil & Propoxyphene \\
\hline Amiodarone & Furosemide & Proton pump inhibitors \\
\hline Amlodipine & Gabapentin & Quinupristin/dalfopristin \\
\hline Ampicillin & Gold & Ranitidine \\
\hline Antivirals & HAART agents & Repaglinide \\
\hline Aspirin & HMG-CoA reductase & Rifampin \\
\hline Atypical antipsychotics & inhibitors & Rifapentine \\
\hline Azathioprine & Ifosfamide & Rivastigmine \\
\hline Bupropion & Indomethacin & Ropinirole \\
\hline Calcitriol & Interferon/ribavirin & Saw palmetto \\
\hline Cannabis & Interleukin-2 & Selective serotonin \\
\hline Capecitabine & Irbesartan & receptor antagonists \\
\hline Carbamazepine & Isoniazid & Sirolimus \\
\hline Ceftriaxone & Isotretinoin & Sodium stibogluconate \\
\hline Cimetidine & Lamotrigine & Somatropin \\
\hline Cisplatin & L-asparaginase & Sulfamethoxazole \\
\hline Clomiphene & Macrolides & Sulfasalazine \\
\hline Codeine & Mefenamic acid & Sumatriptan \\
\hline Colchicine & 6-Mercaptopurine & Tacrolimus \\
\hline Corticosteroids & Mesalamine & Tamoxifen \\
\hline COX-2 inhibitors & Metformin & Tetracyclines \\
\hline Cyclophosphamide & Methimazole & Thiazide diuretics \\
\hline Cyclosporine & Methyldopa & Thrombolytic agents \\
\hline Cyproheptadine & Metronidazole & TNF-alpha inhibitors \\
\hline Cytosine & Mirtazapine & Topiramate \\
\hline Danazol & Montelukast & Trimethoprim- \\
\hline Dapsone & Mycophenolate & sulfamethizole \\
\hline Diazoxide & Nitrofurantoin & Valproic acid \\
\hline Diphenoxylate & NSAIDs & Venlafaxine \\
\hline Dipyridamole & Octreotide & Vincristine \\
\hline Doxercalciferol & Paclitaxel & Voriconazole \\
\hline Doxorubicin & Pegaspargase & Zolmitriptan \\
\hline Ertapenem & Penicillin & \\
\hline
\end{tabular}

*From references 7-11.

ACE indicates angiotensin-converting enzyme; COX, cyclooxygenase; HMG-CoA, 3-hydroxy-3-methylglutaryl coenzyme A; NSAID, nonsteroidal anti-inflammatory drug; TNF, tumor necrosis factor.

\section{HMG-COA REDUCTASE INHIBITORS}

It has been suggested that drug-induced AP by HMG-CoA reductase inhibitors, often referred to as statins, is a class effect $(7,13)$. Case reports of druginduced AP have been published for all of the statins, with rosuvastatin the most recent statin implicated. According to Badalov and colleagues, statins are categorized as class Ia, Ib, III, and IV.

Thisted and colleagues conducted a populationbased, case-control study that evaluated the risk of AP associated with statins (14). The study included 2576 new hospital admissions for AP and 25,817 matched controls. The analysis included "ever before" users, "current" users that filled a prescription within 90 days of diagnosis, "new" users that filled the first prescription within 90 days of diagnosis, and "former" users that had not filled a prescription in $>90$ days from diagnosis. AP was diagnosed in 3.9\% of "ever" users and $2.9 \%$ of controls (adjusted odds ratio, 1.44; $95 \%$ confidence interval [CI], 1.15 to 1.80 ). Both the "current" and "former" users demonstrated an increased risk of developing AP (adjusted odds ratios, 1.26 and 2.02, respectively; $95 \%$ CIs, 0.96 to 1.64 and 1.37 to 2.97 , respectively). Lastly, an increased risk of AP was not noted among "new" users. The investigators concluded that there was not a strong association between statins and AP and instead suggested a possible protective effect. Furthermore, a review of controlled observational studies and case reports published by Singh and Loke concluded that statin-induced AP was both dose-independent and unpredictable (15).

Statin-induced AP can develop from hours to years after initiation of therapy $(14,15)$. The lack of a consistent latency period suggests a possible direct toxic effect to the pancreas and accumulation of a toxic metabolite as possible mechanisms (14). Other mechanisms of action of statin-induced AP are speculated to be associated with rhabdomyolysis, myalgia, and/or metabolism or drug interactions through CYP3A4 $(15,16)$. In several case reports, either myalgia or rhabdomyolysis occurred before development of $\operatorname{AP}(15,16)$. Pravastatin may have fewer case reports of drug-induced AP than other statins because it is not metabolized by CYP3A4 (15). ized angioedema effect within the pancreas. For estrogens and hormone replacement therapy (HRT), arteriolar thrombosis is a theorized mechanism of action.

Published case reports of drug-induced AP exist for at least 40 drugs of the top 200 most prescribed medications. This article reviews data on five drug classes or drugs commonly associated with AP: 3-hydroxy-3-methyl glutaryl coenzyme A (HMG-CoA) reductase inhibitors, ACE inhibitors, estrogens/ HRT, diuretics, highly active antiretroviral therapy (HAART), and valproic acid. Most of these drugs are among the top 200 most prescribed medications for 2007.

\section{ACE INHIBITORS}

The case reports of ACE inhibitor-induced AP involve benazepril, captopril, enalapril, lisinopril, quinapril, and ramipril. ACE inhibitors are categorized as class Ia, III, and IV according to the classification system constructed by Badalov and colleagues.

Eland and colleagues conducted a European case-control study that assessed the risk of AP associated with ACE inhibitors (17). Study participants were 40 to 85 years of age with either a hospital admission for AP or development of AP within 3 
days of admission. Based on 724 cases and 1791 controls, the results indicated that ACE inhibitors were associated with an increased risk of developing AP (adjusted odds ratio, 1.5; 95\% $\mathrm{CI}, 1.1$ to 2.2 ). Furthermore, the study demonstrated that a higher risk of $\mathrm{AP}$ occurred within the first 6 months of initiating therapy (adjusted odds ratio, 3.2; 95\% CI, 1.4 to 7.3 ) and with higher doses of ACE inhibitors (adjusted odds ratio, 3.3; 95\% CI, 0.8 to 14.3 ).

The proposed mechanism of action for ACE inhibitor-induced AP is local angioedema of the pancreatic duct $(17,18)$. ACE inhibitors decrease the degradation of bradykinin, which is linked to the development of angioedema (19). It has also been demonstrated that bradykinins are released during AP, which correlates with the development of increased pancreatic vascular permeability (19). As a result, pancreatic edema can occur and enzymes and other toxic substances can be trapped within the pancreas, leading to pancreatic tissue damage and pancreatitis (19). Lastly, angiotensin II receptors may be important in the regulation of pancreatic secretion and microcirculation (19).

\section{ORAL CONTRACEPTIVES/HRT}

Oral contraceptives and HRT with estrogen with or without progestins have been associated with AP. Badalov and colleagues categorized estrogen-containing agents as class Ib and II.

In a Danish case-control study, the association of HRT and AP was evaluated in 1054 former and current users of HRT (either estrogen alone or estrogen combined with progestin) and 10,540 population controls (20). Cases involved women over the age of 45 years with a first-time hospital discharge for AP; the study excluded patients with a diagnosis of gallstone disease, alcohol-related disease, and inflammatory bowel disease. The results indicated a nonsignificant increased risk of developing $\mathrm{AP}$ in former users of combined estrogen and progestin HRT (adjusted relative risk, 1.6; 95\% CI, 1.0 to 2.5 ). The authors concluded that the increased risk among former users could have been due to either chance or the discontinuation of HRT, which may have led to hypertriglyceridemia, a risk factor for AP. Unfortunately, a limitation of the study was the inability to identify women with hypertriglyceridemia.

There are two proposed mechanisms of action for estrogeninduced AP, both of which are related to the adverse effects of oral contraceptives and HRT. The first mechanism suggests that patients develop hypertriglyceridemia either as a new diagnosis, an exacerbation of an existing hypertriglyceridemia, or a diagnosis of a previously unknown familial hyperlipoproteinemia $(7,12,21-23)$. The second proposed mechanism of action is that estrogens induce a hypercoagulable state, which can lead to pancreatic necrosis $(12,21,23)$.

\section{DIURETICS}

Loop diuretics and hydrochlorothiazides have been implicated in drug-induced AP. Furosemide is a class Ia agent, while thiazide diuretics are class II and III agents (7).

A prospective, case-control study conducted by Bourke and colleagues evaluated medication histories in patients who presented with a first episode of AP (24). Medications taken by these patients included both furosemide and thiazide diuretics. The investigators concluded that patients who took diuretics presented with AP two and a half times more often than those in the control group. Furthermore, patients who developed AP were more likely to be taking thiazide diuretics. However, Eland and colleagues also investigated the risk of AP associated with potassium-sparing, loop, and thiazide diuretics (17). They concluded that there was not an increased risk of AP with loop and thiazide diuretics, and there was a nonsignificant increased risk with potassium-sparing diuretics (adjusted odds ratio, 1.7; 95\% CI, 0.98 to 2.8 ).

Suggested mechanisms of action for furosemide-induced AP include a direct toxic effect to the pancreas, diuretic-induced stimulation of pancreatic secretion, and ischemia $(8,17,19)$. An experimental study demonstrated that a decrease in extracellular fluid volume impairs pancreatic blood flow, which leads to ischemia (22). Two adverse effects of hydrochlorothiazides are hypercalcemia and hyperlipidemia. Hydrochlorothiazides increase calcium bone reabsorption and increase the serum calcium level, which is a risk factor for AP $(22,23)$. Furthermore, hydrochlorothiazides may be involved in the development of hyperparathyroidism, which can lead to hypercalcemia and AP $(18,19)$. Serum triglyceride levels may also increase with the use of hydrochlorothiazides $(22,23)$.

\section{HAART THERAPY}

Patients infected with HIV are 35 to 800 times more likely to develop AP than the general population $(2,25)$. AP is commonly associated with both HIV and use of HAART in HIVpositive patients. The antiretroviral agents linked to AP include protease inhibitors (PI) and nucleoside reverse transcriptase inhibitors (NRTI). Badalov and colleagues categorized four HAART agents as follows: lamivudine and nelfinavir as class $\mathrm{Ib}$ agents, didanosine as a class II agent, and ritonavir as a class IV agent.

In a retrospective cohort study, investigators utilized the Ohio Medicaid claims database to evaluate the risk of AP with various HAART drug regimens (25). Of the 4972 patients who received antiretroviral therapy, 3.2\% developed AP. AP occurred in $4.2 \%$ of newly diagnosed and $2.6 \%$ of previously diagnosed HIV patients. Furthermore, the results of the study indicated that AP occurred in $5.2 \%$ of patients who received didanosine plus other antiretroviral agents, $4.2 \%$ of patients who received a PI plus either an NRTI or a non-nucleoside reverse transcriptase inhibitor (NNRTI), and 3.5\% of patients who received NRTIs combined with NNRTIs. The investigators also identified risk factors for AP, which included advanced HIV disease, preexisting liver disease, increased age, and non-Caucasian race (25).

A potential mechanism of AP is the HIV infection itself, which causes direct inflammation of the pancreas $(2,25)$. HAART drug therapy may also be involved in the development of AP. The antiretroviral therapy may cause either a direct toxic effect on the pancreas or an adverse effect associated with AP. A serious adverse effect of PIs is metabolic disturbance, which includes development of insulin resistance, hyperglycemia, hypercholesterolemia, and hypertriglyceridemia. However, AP has 
been reported in patients who received PIs but did not have elevated triglyceride levels (26). Lastly, in a retrospective study, the investigators concluded that after the introduction of PIs, there was not a significant increase in prevalence of AP (26).

\section{VALPROIC ACID}

Valproic acid-induced AP has been reported since 1979 and is noted to occur more often in children (27). The incidence in the pediatric population has been estimated to be $13 \%$ (27). In 2000, the US Food and Drug Administration issued a black box warning on the potential risk of fatal pancreatitis with the use of valproic acid $(28,29)$. Valproic acid is classified by Badalov and colleagues as a class Ia agent.

A retrospective study conducted by Pellock and colleagues reviewed $\mathrm{AP}$ cases in the valproic acid/divalproex clinical trials database (29). Of 3007 patients who received valproic acid, $0.2 \%$ developed AP. Most patients had other potential etiological risk factors for AP, such as cholelithiasis or other concomitant medications associated with AP. There was no significant difference between the valproic acid-and placebo-treated groups in reference to elevated amylase levels. The authors concluded that valproic acid-induced AP is uncommon and may be associated with the other risk factors present. In contrast to this conclusion of an uncommon incidence of valproic acid-induced AP, Gerstner and colleagues conducted a questionnaire study in Germany and identified 16 documented cases of valproic acid-induced AP over 10 years (27). They concluded that valproic acid-induced AP was underreported.

A population-based, case-control study conducted by Norgaard and colleagues evaluated the risk of AP associated with valproic acid in a Danish population (30). The study included 3083 valproic acid users (cases) and 30,830 other antiepileptic users (controls) diagnosed with AP from 1998 to 2003. Present use of valproic acid was defined as receiving at least one prescription within 90 days of admission for AP. Past use was defined as receiving a valproic acid prescription within 91 to 365 days of AP diagnosis. The results demonstrated that among the cases, AP occurred in $0.52 \%$ of present and $0.13 \%$ of past valproic acid users. The occurrence of AP was higher among present and past users of valproic acid than among present and past users of other antiepileptics. The difference between past users of valproic acid or other antiepileptics was greater than among present users (adjusted odds ratios, 2.6 and 1.8, respectively; $95 \%$ CIs, 0.8 to 8.7 and 1.1 to 3.0 , respectively). The investigators concluded that valproic acid was associated with an increased risk of AP but hypothesized that other risk factors could be involved in the development of AP.

Valproic acid-induced AP typically occurs within the first year of treatment, and risk increases with higher doses (29). The proposed mechanisms of action of valproic acid-induced AP are a direct toxic effect of free radicals on the pancreatic tissue and a depletion of superoxide dismutase, catalase, and glutathione peroxidase (27). Based on results of their case series of valproic acid-induced AP, Sinclair and colleagues suggested that an idiosyncratic drug reaction may be a mechanism, especially in patients with a history of drug sensitivity (31).

\section{CONCLUSION}

Case reports continue to be published that reaffirm medications known to cause drug-induced AP and implicate new medications. A substantial number of drugs commonly prescribed in the USA are known to cause AP, but there is limited understanding of the mechanisms involved. Many of the hypothesized mechanisms of action are related to the adverse effects of the drug that are risk factors for AP. These effects include hypertriglyceridemia or hypercalcemia caused by medications such as oral contraceptives, HRT, or hydrochlorothiazides. Other mechanisms of drug-induced AP may be related to the mechanism of action of the drug, such as with ACE inhibitors and furosemide. The greatest challenge to understanding the cause of drug-induced AP is the ability to rule out other causes. Patients diagnosed with a drug-induced AP may have unknown underlying risk factors, and initiation of the medication could exacerbate the patient's risk. It is prudent for clinicians to consider high-risk medications associated with AP in patients presenting with an unknown etiology.

\section{Acknowledgments}

Erin Sears, PharmD, contributed both expertise and guidance in preparation of this review.

1. Gardner TB, Berk BS, Yakshe P. Acute pancreatitis. eMedicine, updated July 2006. Available at http://www.emedicine.com/med/topic1720.htm; accessed September 30, 2007.

2. Sekimoto M, Takada T, Kawarada Y, Hirata K, Mayumi T, Yoshida M, Hirota M, Kimura Y, Takeda K, Isaji S, Koizumi M, Otsuki M, Matsuno S; JPN. JPN guidelines for the management of acute pancreatitis: epidemiology, etiology, natural history, and outcome predictors in acute pancreatitis. J Hepatobiliary Pancreat Surg 2006;13(1):10-24.

3. Lankisch PG, Droge M, Gottesleben F. Drug induced acute pancreatitis: incidence and severity. Gut 1995;37(4):565-567.

4. Berardi RR, Montgomery PA. Pharmacotherapy: a pathophysiologic approach. In DiPiro JT, Talbert RL, Yee GC, Matzke GR, Wells BG, Posey LM, eds. Pancreatitis, 6th ed. New York: McGraw-Hill, 2005:721-736.

5. Neoptolemos JP, Raraty M, Finch M, Sutton R. Acute pancreatitis: the substantial human and financial costs. Gut 1998;42(6):886-891.

6. Soran A, Chelluri L, Lee KK, Tisherman SA. Outcome and quality of life of patients with acute pancreatitis requiring intensive care. J Surg Res 2000;91(1):89-94.

7. Badalov N, Baradarian R, Iswara K, Li J, Steinberg W, Tenner S. Druginduced acute pancreatitis: an evidence-based review. Clin Gastroenterol Hepatol 2007;5(6):648-661.

8. Denker PS, Dimarco PE. Exenatide (exendin-4)-induced pancreatitis: a case report. Diabetes Care 2006;29(2):471.

9. Ahmad I, Ruby E, Usman H, Hotiana M, Hussain M, Rahman F. Ezetimibe-induced acute pancreatitis. South Med J 2007;100(4):409-410.

10. Jibrin I, Erinle A, Saidi A, Aliyu ZY. Saw palmetto-induced pancreatitis. South Med J 2006;99(6):611-612.

11. Trivedi CD, Pitchumoni CS. Drug-induced pancreatitis: an update. $J$ Clin Gastroenterol 2005;39(8):709-716.

12. Underwood TW, Frye CB. Drug-induced pancreatitis. Clin Pharm 1993;12(6):440-448.

13. Singh S, Nautiyal A, Dolan JG. Recurrent acute pancreatitis possibly induced by atorvastatin and rosuvastatin. Is statin induced pancreatitis a class effect? JOP 2004;5(6):502-504.

14. Thisted H, Jacobsen J, Munk EM, Norgaard B, Friis S, McLaughlin JK, Sorensen HT, Johnsen SP. Statins and the risk of acute pancreatitis: a population-based case-control study. Aliment Pharmacol Ther 2006;23(1):185190 
15. Singh S, Loke YK. Statins and pancreatitis: a systematic review of observational studies and spontaneous case reports. Drug Saf2006;29(12):11231132.

16. Johnson JL, Loomis IB. A case of simvastatin-associated pancreatitis and review of statin-associated pancreatitis. Pharmacotherapy 2006;26(3):414422.

17. Eland IA, Sundstrom A, Velo GP, Andersen M, Sturkenboom MC, Langman MJ, Stricker BH, Wiholm B; EDIP Study Group of the European Pharmacovigilance Research Group. Antihypertensive medication and the risk of acute pancreatitis: the European case-control study on drug-induced acute pancreatitis (EDIP). Scand J Gastroenterol 2006;41(12):14841490 .

18. Kanbay M, Selcuk H, Yilmaz U, Boyacioglu S. Recurrent acute pancreatitis probably secondary to lisinopril. South Med J 2006;99(12):1388-1389.

19. Griesbacher T. Kallikrein-kinin system in acute pancreatitis: potential of $\mathrm{B}_{2}$-bradykinin antagonists and kallikrein inhibitors. Pharmacology 2000;60(3):113-120.

20. Tetsche MS, Jacobsen J, Norgaard M, Baron JA, Sorensen HT. Postmenopausal hormone replacement therapy and risk of acute pancreatitis: a population-based case-control study. Am J Gastroenterol 2007;102(2):275278.

21. Banerjee AK, Patel KJ, Grainger SL. Drug-induced acute pancreatitis. A critical review. Med Toxicol Adverse Drug Exp 1989;4(3):186-198.

22. Frick TW, Speiser DE, Bimmler D, Largiader F. Drug-induced acute pancreatitis: further criticism. Dig Dis 1993;11(2):113-132.
23. Runzi M, Layer P. Drug-associated pancreatitis: facts and fiction. Pancreas 1996;13(1):100-109.

24. Bourke JB, Mead GM, McIllmurray MB, Langman MJS. Drug-associated primary acute pancreatitis. Lancet 1978; 1(8066):706-708.

25. Guo JJ, Jang R, Louder A, Cluxton RJ. Acute pancreatitis associated with different combination therapies in patients infected with human immunodeficiency virus. Pharmacotherapy 2005;25(8):1044-1054.

26. Bush ZM, Kosmiski LA. Acute pancreatitis in HIV-infected patients: are etiologies changing since the introduction of protease inhibitor therapy? Pancreas 2003;27(1):e1-e5.

27. Gerstner T, Busing D, Bell N, Longin E, Kasper JM, Klostermann W, Hebing B, Hanefeld F, Eckel U, Hoffmann R, Bettendorf U, Weidner B, Wiemer-Kruel A, Brockmann K, Neumann FW, Sandrieser T, Wolff M, Konig S. Valproic acid-induced pancreatitis: 16 new cases and a review of the literature. J Gastroenterol 2007;42(1):39-48.

28. Depakote (divalproex) [package insert]. North Chicago, IL: Abbott Laboratories, 2006.

29. Pellock JM, Wilder BJ, Deaton R, Sommerville KW. Acute pancreatitis coincident with valproate use: a critical review. Epilepsia 2002;43(11):14211424.

30. Norgaard M, Jacobsen J, Ratanajamit C, Jepsen P, McLaughlin JK, Pedersen L, Sorensen HT. Valproic acid and risk of acute pancreatitis: a population-based case-control study. Am J Ther 2006;13(2):113-117.

31. Sinclair DB, Berg M, Breault R. Valproic acid-induced pancreatitis in childhood epilepsy: case series and review. J Child Neurol 2004;19(7):498_ 502 . 